ethical objections to the "medicalization" of behavior and learning. (Millichap JG. Attention Deficit Hyperactivity and Learning Disorders. Chicago, PNB Publishers, 2001, p9). For parent training to be effective, the coaching must be specific and constructive regarding behavioral management techniques. A nondirect method of parent counseling and support, without training in behavioral strategies, is largely ineffective. The completion of a weekly behavior diary by the parent is an essential adjunct to regular reviews of results of intervention and identification of problems.

In the recent NIMH Collaborative Multisite Multimodal Treatment Study (MTS) of grade school children with ADHD, intensive psychosocial intervention alone was much less effective than psychostimulant medication and no more effective than routine community-based care. In combination with medication, psychosocial intervention was only slightly additive in benefit (Jensen PS et al. Arch Gen Psychiatry 1999;56:1073-1086; see Ped Neur Briefs Jan 2000;14:3-4). Parent training techniques are time consuming, requiring frequent follow-up and reinforcement. The practical disadvantages of PT compared to medical treatment would need to be addressed, for this form of intervention to be generally successful. For children under 5 years of age, when treatment with stimulant medication is not appropriate, a trial of PT is especially indicated.

In the UK where the above study was conducted, the recognition and treatment of ADHD with medication is not as readily accepted as in the US (Taylor E. Arch Gen Psychiatry 1999;56:1097-1099). Many UK parents will opt for no therapy or for behavioral training, despite the obvious superior benefits of methylphenidate demonstrated in the MTS study.

\title{
CEREBRAL GRAY MATTER VOLUME REDUCTIONS IN DYSLEXICS
}

Brain MR images were compared in 16 right-handed men (mean age, 24 years; range 18 to 40 ) with dyslexia and 14 control subjects using a voxel-based analysis, at Stanford University School of Medicine, CA. Evidence of decreases in gray matter in dyslexics was most notable in the left temporal lobe, especially the left posterior superior temporal gyrus (STG), the temporoparietooccipital juncture bilaterally, and the frontal lobe, caudate, thalamus, and cerebellum. Morphological variations in brain structure affecting several brain regions may explain the neuroanatomical basis of dyslexia. (Brown WE, Eliez S, Menon V et al. Preliminary evidence of widespread morphological variations of the brain in dyslexia. Neurology March (2 of 2) 2001;56:781-783). (Reprints: Dr AL Reiss, Department of Psychiatry and Behavioral Sciences, Stanford University School of Medicine, 401 Quarry Rd, Stanford, CA 94305).

COMMENT. This study confirms previous MR evidence of decreases in the left temporal lobe volume in dyslexic subjects, and further localizes the deficit to the STG and left inferior, middle, and mesial temporal regions. The decreases are global in distribution and affect subcortical as well as cortical gray matter and also, the cerebellum.

In a Yale University study (Schultz RT et al. Ann Neurol 1994;35:732-742), the influence of age, sex and overall brain size on the measurement of MRI brain volume changes in dyslexia was stressed. Analyses that controlled for these variables failed to confirm smaller left hemisphere structures previously reported in dyslexia. Studies involving changes in the corpus callosum are reported from other centers. (see Progress in Pediatric Neurology III, PNB Publ, 1997;pp269-270; and Ped Neur Briefs Jan 2001;15:1; for further comment on the neurological basis of developmental dyslexia). 